\author{
Wolfgang Huber \\ Sebastian Mair \\ Simon Q. Götz \\ Julia Tschirdewahn \\ Johanna Siegel \\ Roland M. Schmid \\ Bernd Saugel
}

\section{Extravascular lung water and its association with weight, height, age, and gender: a study in intensive care unit patients}

Received: 20 May 2012

Accepted: 23 October 2012

Published online: 18 November 2012

(C) The Author(s) 2012. This article is published with open access at Springerlink.com
W. Huber · S. Mair · S. Q. Götz ·

J. Tschirdewahn · J. Siegel ·

R. M. Schmid · B. Saugel (ه)

II. Medizinische Klinik und Poliklinik,

Klinikum rechts der Isar der Technischen

Universität München, Ismaninger Straße 22,

81675 Munich, Germany

e-mail: bcs.muc@gmx.de

Tel.: +49-89-41405478
Abstract Purpose: With regard to large inter-individual variability of height, body weight (BW), and age, several hemodynamic parameters are adjusted for biometric data. This also applies to extravascular lung water (EVLW), which traditionally was indexed to actual BW (BW-act) resulting in EVLW-index (EVLWI; i.e., EVLWI-act). Since indexation to BW-act might inappropriately diminish EVLWI-act in obese patients, the indexation has been changed to predicted BW (BW-pred) resulting in EVLWI-pred. BW-pred is a weight estimation formula calculated from height and gender that has not been derived from populationbased data. The aim of the study was to investigate the independent association of biometric data with EVLW. Methods: We analyzed a hemodynamic monitoring database including 3,691 transpulmonary thermodilution-derived EVLW measurements (234 consecutive patients; intensive care unit of a university hospital). We performed univariate and multivariate analyses regarding the association of biometric data with the first EVLW measurement and the mean EVLW value of each patient. Results: In univariate analysis, the first EVLW significantly correlated with height $(r=0.254 ; p<0.001)$, but neither with age nor BW-act. Similar findings were made in the analysis of the patients' EVLW means of all measurements ("one point per patient"). In multivariate analysis (primary endpoint), including BW-act, height, age, and gender, only height was independently associated with EVLW, with each centimeter of height increasing the first measurement of EVLW by $6.882 \mathrm{~mL}$ $(p<0.001)$ and mean EVLW by $6.727 \mathrm{~mL}(p<0.001)$. Conclusions: Height is the only biometric parameter independently associated with the first and mean EVLW. In adult patients, EVLW should be indexed to height.

Keywords Extravascular lung water - Extravascular lung water index - Hemodynamic monitoring . Transpulmonary thermodilution . Body weight · Biometry

\section{Introduction}

Extravascular lung water (EVLW) estimates fluid in the pulmonary interstitial and alveolar spaces. EVLW can be assessed using single-indicator transpulmonary thermodilution (TPTD) and is associated with pulmonary function and mortality [1-11].

EVLW traditionally was indexed to actual body weight (BW) (BW-act) resulting in extravascular lung water index (EVLWI) (EVLWI-act). However, especially 
in obese patients, indexation of EVLW to BW-act has been questioned [5-8]. Based on better mortality prediction by EVLWI indexed to BW-pred (EVLWI-pred) in some studies [5-7], both commercially available TPTD devices at present provide EVLWI-pred. However, data on appropriate indexation of EVLW in terms of prediction of mortality or correlation with $\mathrm{PaO}_{2} / \mathrm{FiO}_{2}$ are not consistent $[5-8,12]$.

When evaluating the appropriateness of different EVLW indexations, mortality is an obvious endpoint. However, mortality is multifactorial and prone to confounding factors and direct association with biometric data (age, BW) [13, 14]. Similar considerations apply to $\mathrm{PaO}_{2} / \mathrm{FiO}_{2}$ ratios. Therefore, multiple regression analysis regarding the independent association of EVLW with biometric data might be a more systematical approach. Therefore, we analyzed a database of EVLW measurements regarding the independent association of BW-act, height, age, and gender with un-indexed EVLW.

\section{Materials and methods}

The study was approved by the institutional review board. We analyzed data of a prospectively maintained hemodynamic database including 4,913 TPTD measurements in 289 consecutive patients discharged from our ICU between October 2008 and December 2010. Since EVLW values might be altered when using inferior vena cava access for TPTD [15], only EVLW measurements using superior vena cava access for indicator injection were analyzed (3,691 EVLW determinations in 234 patients).

TPTD was performed as described previously using the PiCCO device (Pulsion Medical Systems, Munich, Germany) $[16,17]$.

The primary endpoint was to identify biometric parameters independently associated with un-indexed EVLW using multivariate regression analysis preceded by univariate analysis. The comparison of mean values of EVLWI-act and EVLWI-pred in all patients and body mass index (BMI) subgroups of patients were secondary endpoints.

Statistical analyses were performed using IBM SPSS Statistics 19 (SPSS Inc., Chicago, IL, USA).

EVLW was indexed to different weight formulas (Table 1). Distributions of normal $(\leq 7 \mathrm{~mL} / \mathrm{kg})$, slightly elevated $(7<$ EVLWI $\leq 10 \mathrm{~mL} / \mathrm{kg})$, and markedly elevated EVLWI $(>10 \mathrm{~mL} / \mathrm{kg})$ depending on different indexations were calculated for all patients and for BMI subgroups. Percentages were calculated on the basis of the measurements with valid data. The proportion of normal EVLWI values indexed to either BW-act or BW-pred was compared using the $\chi^{2}$ test. For comparison of mean EVLWI values according to different weight-based

Table 1 Patients characteristics and comparison of EVLWI according to different indexations (all measurements)

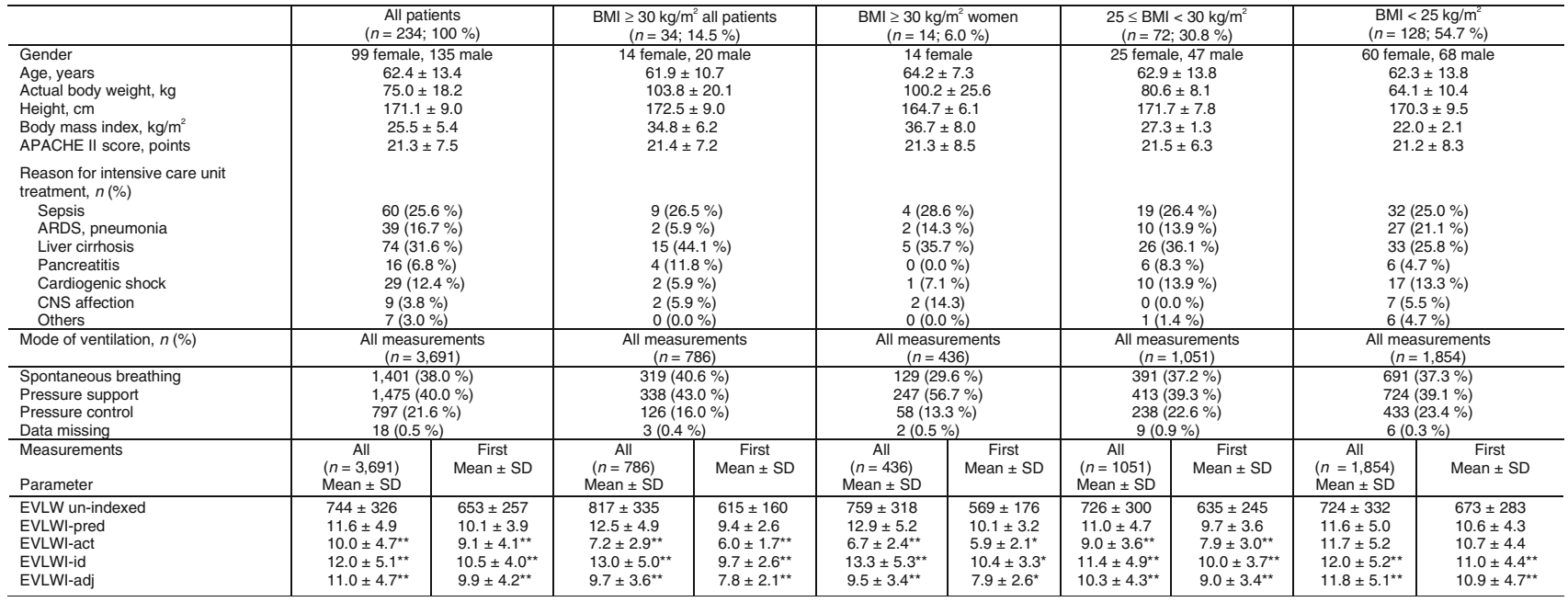

Weight correction formulas were used as follows:

Predicted body weight (BW-pred) $(\mathrm{kg})$ : Male: $50+0.91 \times$ [height (cm) -152.4$]$; Female: $45.5+0.91 \times$ [height $(\mathrm{cm})-152.4]$

Ideal body weight $(\mathrm{BW}-\mathrm{id})(\mathrm{kg})$ : Male: [height $(\mathrm{cm})-100] \times 0.9$; Female: [height $(\mathrm{cm})-100)$ ] $\times 0.85$

Adjusted body weight (BW-adj) (kg): Male: $\mathrm{BW}-\mathrm{id}+(\mathrm{BW}$-act - BW-id) $\times 0.4$; Female: BW-id + (BW-act - BW-id $) \times 0.4$ $B M I$ body mass index, ARDS acute respiratory distress syndrome, $C N S$ central nervous system, EVLW extravascular lung water,
EVLWI-pred extravascular lung water index indexed to predicted body weight, $E V L W I$-act extravascular lung water index indexed to actual body weight, EVLWI-id extravascular lung water index indexed to ideal body weight, EVLWI-adj extravascular lung water index indexed to adjusted body weight

$* p<0.05$ vs. EVLWI-pred (assuming EVLWI-pred to be the standard indexation); ** $p<0.001$ vs. EVLWI-pred (assuming EVLWI-pred to be the standard indexation) 
Fig. 1 Mean values of extravascular lung water index according to different indexations (all measurements). Bar graph showing mean extravascular lung water index (EVLWI) values according to different indexations in all patients and in women with body mass index $(\mathrm{BMI}) \geq 30$ $\mathrm{kg} / \mathrm{m}^{2}$. Extravascular lung water index indexed to predicted body weight (EVLWI-pred), actual body weight (EVLWI-act), ideal body weight (EVLWI-id), or adjusted body weight (EVLWI-adj)

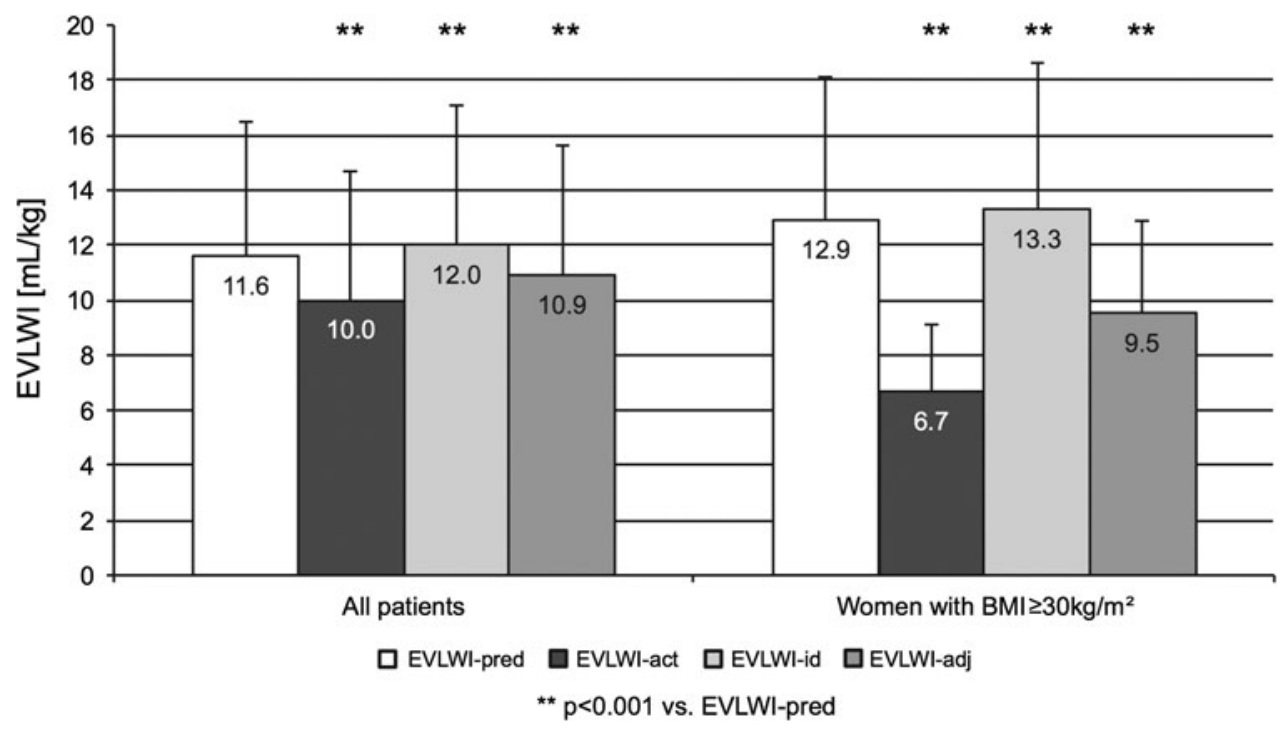

formulas, we used the Wilcoxon test for paired samples. For group comparisons between female and male patients regarding continuous variables, the Mann-Whitney $U$ test was performed.

To eliminate the effect of different numbers of measurements in different individuals, we performed univariate and multivariate analyses based on the first EVLWI measurement of each patient and separately based on the mean EVLWI value of each patient ("one point per patient").

For ex-post group comparisons of markers of lung injury between patients within the four $25 \%$ percentiles of height and $\mathrm{BW}$ we used the Kruskal-Wallis test. We performed receiver operating characteristic (ROC) analysis to evaluate the discriminative power of differently indexed EVLW with regard to " $\mathrm{PaO}_{2} / \mathrm{FiO}_{2}<200 \mathrm{mmHg}$.

\section{Results}

Patients' basic characteristics are shown in Table 1.
Comparison of EVLWI values according to different indexations

In the totality of patients, mean EVLWI-pred (all measurements) was significantly higher than EVLWI-act $(11.6 \pm 4.9$ vs. $10.0 \pm 4.7 \mathrm{~mL} / \mathrm{kg} ; p<0.001)$ (Table 1 , Fig. 1). This difference was even more pronounced in patients with $\mathrm{BMI} \geq 30 \mathrm{~kg} / \mathrm{m}^{2} \quad(12.5 \pm 4.9$ vs. $7.2 \pm$ $2.9 \mathrm{~mL} / \mathrm{kg} ; \quad p<0.001)$ and in patients with $25 \leq$ $\mathrm{BMI}<30 \mathrm{~kg} / \mathrm{m}^{2} \quad(11.0 \pm 4.7 \quad$ vs. $9.0 \pm 3.6 \mathrm{~mL} / \mathrm{kg}$; $p<0.001)$. The difference between EVLWI-pred and EVLWI-act was most pronounced in women with $\mathrm{BMI} \geq 30 \mathrm{~kg} / \mathrm{m}^{2}(12.9 \pm 5.2$ vs. $6.7 \pm 2.4 \mathrm{~mL} / \mathrm{kg} ; p<$ 0.001 (Fig. 1). Only in patients with BMI $<25 \mathrm{~kg} / \mathrm{m}^{2}$ there was no significant difference between EVLWI-pred $(11.6 \pm 5.0 \mathrm{~mL} / \mathrm{kg})$ and EVLWI-act $(11.7 \pm 5.2 \mathrm{~mL} / \mathrm{kg})$.

Similar results were found when analyzing the first EVLW measurement in each patient (Table 1).

The use of BW-pred or BW-act for indexation of EVLW resulted in a markedly different distribution of EVLWI values within the normal range, slightly elevated EVLWI values, and markedly elevated EVLWI (all patients and BMI subgroups) (Table 2). The percentage of

Table 2 Distribution of normal, slightly elevated, and markedly elevated EVLWI values according to different indexations (all measurements)

\begin{tabular}{|c|c|c|c|c|c|c|}
\hline \multirow[t]{3}{*}{ EVLWI-pred (mL/kg) } & \multicolumn{6}{|c|}{ EVLWI-act (mL/kg) } \\
\hline & \multicolumn{3}{|c|}{ All measurements } & \multicolumn{3}{|c|}{ Measurements in patients with $\mathrm{BMI} \geq 30 \mathrm{~kg} / \mathrm{m}^{2}$} \\
\hline & $\leq 7$ & $7<$ EVLWI $\leq 10$ & $>10$ & $\leq 7$ & $7<$ EVLWI $\leq 10$ & $>10$ \\
\hline $\begin{array}{l}\leq 7 \\
7<\mathrm{EVLWI} \leq 10 \\
>10\end{array}$ & $\begin{array}{c}495(13.4 \%) \\
379(10.3 \%) \\
183(5.0 \%)\end{array}$ & $\begin{array}{c}99(2.7 \%) \\
710(19.2 \%) \\
405(11.0 \%)\end{array}$ & $\begin{array}{c}0(0.0 \%) \\
147(4.0 \%) \\
1,273(34.5 \%)\end{array}$ & $\begin{array}{c}57(7.3 \%) \\
230(29.3 \%) \\
183(23.3 \%)\end{array}$ & $\begin{aligned} 0 & (0.0 \%) \\
33 & (4.2 \%) \\
184 & (23.4 \%)\end{aligned}$ & $\begin{array}{c}0(0.0 \%) \\
0(0.0 \%) \\
99(12.6 \%)\end{array}$ \\
\hline
\end{tabular}

EVLWI extravascular lung water index, EVLWI-act extravascular lung water index indexed to actual body weight, EVLWI-pred extravascular lung water index indexed to predicted body weight, $B M I$ body mass index 
normal measurements $(\leq 7 \mathrm{~mL} / \mathrm{kg})$ was significantly different for all subgroups when comparing EVLWI-pred and EVLWI-act. Normal values of EVLWI were significantly more frequent for EVLWI-act in the totality of patients $(1,057 / 3,691(28.6 \%)$ vs. 594/3,691 (16.1\%); $p<0.001)$ as well as in patients with BMI $\geq 30 \mathrm{~kg} / \mathrm{m}^{2}$ (470/786 $(59.8 \%)$ vs. $57 / 786(7.3 \%) ; p<0.001)$, women with $\mathrm{BMI} \geq 30 \mathrm{~kg} / \mathrm{m}^{2}(306 / 436(70.2 \%)$ vs. $28 / 436(6.4 \%)$; $p<0.001)$, and patients with $25 \leq \mathrm{BMI}<30 \mathrm{~kg} / \mathrm{m}^{2}(200 /$ $1,051(19.0 \%)$ vs. $323 / 1,051(30.7 \%) ; p<0.001)$. Normal values of EVLWI were less frequent for EVLWI-act compared to EVLWI-pred in patients with BMI $<25 \mathrm{~kg} / \mathrm{m}^{2}$ $(264 / 1,854(14.2 \%)$ vs. $337 / 1,854(18.2 \%) ; p=0.001)$.

In the totality of patients, $1,213 / 3,691(32.9 \%) \mathrm{EV}$ LWI measurements would have been classified in different categories when using the different indexations (Table 2). Again, this was more pronounced in obese patients (BMI $\geq 30 \mathrm{~kg} / \mathrm{m}^{2}$ ) with 597/786 $(76.0 \%)$ measurements being differently classified by EVLWI-pred and EVLWI-act.

\section{Association of un-indexed EVLW with biometric data}

In univariate analysis, the first EVLW measurement in each patient significantly correlated with height $(r=0.254$; $p<0.001)$, but neither with age $(r=-0.001 ; p=0.994)$ nor BW-act ( $r=0.102 ; p=0.119)$. In comparison to male patients, female patients had a statistically significantly lower EVLW $(602 \pm 241$ vs. $690 \pm 263 \mathrm{~mL} ; p<0.001)$. However, height $(164.0 \pm 6.4$ vs. $176.3 \pm 6.7 \mathrm{~cm} ; p<0.001)$ and BW-act $(67.9 \pm 19.0$ vs. $80.1 \pm 15.8 \mathrm{~kg} ; p<0.001)$ were also significantly lower in women compared to men.

Similar findings were made in the analysis of the patients' mean EVLW measurements. In univariate analysis, mean EVLW significantly correlated with height $(r=$ $0.255 ; p<0.001)$, but not with age $(r=0.006 ; p=0.932)$. There was a weak but significant correlation between mean EVLW and BW-act ( $r=0.152 ; p=0.020)$.

According to multivariate regression analysis (primary endpoint), among these biometric data, only height was independently associated with the first EVLW measurement in each patient, with each centimeter of height increasing EVLW by $6.882 \mathrm{~mL} \quad(95 \%$ confidence interval, 3.282-10.482; $p<0.001)$. Similarly, means of all EVLW measurements in individual patients were significantly and independently associated with height, with each centimeter of height increasing mean EVLW by $6.727 \mathrm{~mL}(95 \%$ confidence interval, 3.337-10.117 $\mathrm{mL} ; p<0.001$ ).

\section{Ex post statistical analysis}

To preclude that the association of height and EVLW was due to a disproportional distribution of pulmonary impairment in patients with different height, we performed ex post analysis regarding the distribution of lung injury markers among patients within four $25 \%$ percentiles of height. $\mathrm{PaO}_{2} / \mathrm{FiO}_{2}$ was available for only 661 EVLW measurements in 76 patients. There was no statistically significant disproportional distribution of $\mathrm{PaO}_{2} /$ $\mathrm{FiO}_{2}$ between patients with different height $(p=0.498)$. Similar results were obtained for the distribution of lung injury score (without chest X-ray) $(p=0.177)$ and oxygenation index $(p=0.316)$.

To preclude that obesity itself might influence pulmonary impairment we analyzed the impact of different BMI categories (four $25 \%$ percentiles and $\mathrm{BMI}<25$, $25 \leq \mathrm{BMI}<30$, and $\mathrm{BMI} \geq 30 \mathrm{~kg} / \mathrm{m}^{2}$ ) on lung injury markers. Again, there were no hints of an association of BMI with $\mathrm{PaO}_{2} / \mathrm{FiO}_{2}$, lung injury score, or oxygenation index.

ROC analysis evaluating the discriminative power of differently indexed EVLW with regard to " $\mathrm{PaO}_{2}$ / $\mathrm{FiO}_{2}<200 \mathrm{mmHg}$ " demonstrated that EVLWI-height provided a higher ROC area under the curve (AUC) [0.771 (95\% confidence interval, 0.734-0.808)] compared with EVLWI-pred $[0.753 \quad(95 \%$ confidence interval, 0.715-0.791)] and EVLWI-act [0.708 (95\% confidence interval, 0.669-0.747)].

\section{Discussion}

In this study, different weight-based indexations of EVLW resulted in markedly different EVLWI values. Indexation of EVLW to BW-pred and BW-act also resulted in significantly different classifications of the EVLWI measurements as normal, moderately, or markedly elevated. In multivariate regression analysis only height was independently associated with EVLW.

At first glance, these findings might be surprising, since at present $\mathrm{BW}$-act or $\mathrm{BW}$-pred is recommended for indexation. However, expert recommendations on the measurement of lung volumes clearly state that "lung volumes are related to body size, with standing height being the most important factor" [18]. In addition, the formulas for calculation of BW-pred and BW-id do not account for any weight at all, but simply derive BW from height corrected for gender and a constant [19]. While our data support the use of height for indexation of EVLW, our analyses do not demonstrate that gender is independently associated with EVLW. This might explain data from a recent study in which EVLW-pred (including gender) performed worse than EVLWI-act (not correcting for gender) [8].

Several limitations of our study have to be mentioned. This was a single center study in a general ICU with predominantly Caucasian medical patients, which also applies for the majority of previous data [20]. Furthermore, some analyses were ex post analyses with data available only in a limited number of patients. Finally, the explanatory content of height might be considered low with a coefficient of correlation $r=0.254$. However, in general, absolute values 
of EVLW result from both pulmonary pathophysiology as well as biometric data. The impact of biometry might be underestimated in our collective with a high proportion of patients with markedly impaired pulmonary function.

\section{Conclusions}

In 234 adult ICU patients, height was the only biometric parameter independently associated with the first and mean EVLW measurements based on TPTD. Therefore, in adult patients, EVLW should be indexed to height. This results in the unit $(\mathrm{mL} / \mathrm{cm})$ for EVLWI-height.
Acknowledgments We thank Veit Phillip, Caroline Schultheiss, Josef Höllthaler, Philipp Thies, Ulrich Mayr, Sebastian Noe, Henrik Einwächter, Matthias Treiber, and Andrea Herrmann for data acquisition and interpretation.

Conflicts of interest Wolfgang Huber collaborates with Pulsion Medical Systems as a member of the medical advisory board. All other authors have no conflict of interest to disclose.

Open Access This article is distributed under the terms of the Creative Commons Attribution Noncommercial License which permits any noncommercial use, distribution, and reproduction in any medium, provided the original author(s) and the source are credited.

\section{References}

1. Eisenberg PR, Hansbrough JR, Anderson D, Schuster DP (1987) A prospective study of lung water measurements during patient management in an intensive care unit Am Rev Respir Dis 136:662-668

2. Sakka SG, Klein M, Reinhart K, MeierHellmann A (2002) Prognostic value of extravascular lung water in critically ill patients. Chest 122:2080-2086

3. Fernandez-Mondejar E, Castano-Perez J, Rivera-Fernandez R, ColmeneroRuiz M, Manzano F, Perez-Villares J, de la Chica R (2003) Quantification of lung water by transpulmonary thermodilution in normal and edematous lung. J Crit Care 18:253-258

4. Martin GS, Eaton S, Mealer M, Moss M (2005) Extravascular lung water in patients with severe sepsis: a prospective cohort study. Crit Care 9:R74-R82

5. Berkowitz DM, Danai PA, Eaton S, Moss M, Martin GS (2008) Accurate characterization of extravascular lung water in acute respiratory distress syndrome. Crit Care Med 36:1803-1809

6. Phillips CR, Chesnutt MS, Smith SM (2008) Extravascular lung water in sepsis-associated acute respiratory distress syndrome: indexing with predicted body weight improves correlation with severity of illness and survival. Crit Care Med 36:69-73

7. Craig TR, Duffy MJ, Shyamsundar M, McDowell C, McLaughlin B, Elborn JS, McAuley DF (2010) Extravascular lung water indexed to predicted body weight is a novel predictor of intensive care unit mortality in patients with acute lung injury. Crit Care Med 38:114-120
8. Chew MS, Ihrman L, During J, Bergenzaun L, Ersson A, Unden J, Ryden J, Akerman E, Larsson M (2012) Extravascular lung water index improves the diagnostic accuracy of lung injury in patients with shock. Crit Care 16:R1

9. Tagami T, Kushimoto S, Yamamoto $\mathrm{Y}$, Atsumi T, Tosa R, Matsuda K, Oyama R, Kawaguchi T, Masuno T, Hirama H, Yokota H (2010) Validation of extravascular lung water measurement by single transpulmonary thermodilution: human autopsy study. Crit Care 14:R162

10. Letourneau JL, Pinney J, Phillips CR (2012) Extravascular lung water predicts progression to acute lung injury in patients with increased risk. Crit Care Med 40:847-854

11. Michard F, Fernandez-Mondejar E, Kirov MY, Malbrain M, Tagami T (2012) A new and simple definition for acute lung injury. Crit Care Med 40:1004-1006

12. Mallat J, Pepy F, Lemyze M, Barrailler S, Gasan G, Tronchon L, Thevenin D (2012) Extravascular lung water indexed or not to predicted body weight is a predictor of mortality in septic shock patients. J Crit Care 27:376-383

13. Akinnusi ME, Pineda LA, El Solh AA (2008) Effect of obesity on intensive care morbidity and mortality: a metaanalysis. Crit Care Med 36:151-158

14. O'Brien JM Jr, Phillips GS, Ali NA, Lucarelli M, Marsh CB, Lemeshow S (2006) Body mass index is independently associated with hospital mortality in mechanically ventilated adults with acute lung injury. Crit Care Med 34:738-744
15. Saugel B, Umgelter A, Schuster T, Phillip V, Schmid RM, Huber W (2010) Transpulmonary thermodilution using femoral indicator injection: a prospective trial in patients with a femoral and a jugular central venous catheter. Crit Care 14:R95

16. Huber W, Umgelter A, Reindl W, Franzen M, Schmidt C, von Delius S, Geisler F, Eckel F, Fritsch R, Siveke J, Henschel B, Schmid RM (2008) Volume assessment in patients with necrotizing pancreatitis: a comparison of intrathoracic blood volume index, central venous pressure, and hematocrit, and their correlation to cardiac index and extravascular lung water index. Crit Care Med 36:2348-2354

17. Sakka SG, Ruhl CC, Pfeiffer UJ, Beale R, McLuckie A, Reinhart K, Meier-

Hellmann A (2000) Assessment of cardiac preload and extravascular lung water by single transpulmonary thermodilution. Intensive Care Med 26:180-187

18. Wanger J, Clausen JL, Coates A, Pedersen OF, Brusasco V, Burgos F, Casaburi R, Crapo R, Enright P, van der Grinten CP, Gustafsson P, Hankinson J, Jensen R, Johnson D, Macintyre N, McKay R, Miller MR, Navajas D, Pellegrino R, Viegi G (2005) Standardisation of the measurement of lung volumes. Eur Respir J 26:511-522

19. Pai MP, Paloucek FP (2000) The origin of the "ideal" body weight equations. Ann Pharmacother 34:1066-1069

20. Stocks J, Quanjer PH (1995) Reference values for residual volume, functional residual capacity and total lung capacity. ATS workshop on lung volume measurements. Official statement of the European Respiratory Society. Eur Respir J 8:492-506 